\title{
Detecting the Effects of Management Regime Shifts in Dynamic Environments Using Multi-population State-space Models
}

\author{
Matthew R. Falcy and Erik Suring
}

Oregon Department of Fish and Wildlife, Corvallis Research Lab, 28655 Highway 34, Corvallis, OR, 97333, USA

Keywords: Regime shift, nonstationary, population dynamics, relative reproductive success, viability, coho

Detecting the effectiveness of management actions intended to increase the abundance of threatened or exploited species can help resolve uncertainties about cost-effective management tactics. However, the complexity of ecological systems can make it difficult to identify important factors causing change in population abundance. This difficulty extends from detecting naturally-caused ecosystem regime shifts to management induced regime shifts and the attendant change in population dynamics parameters. The adult abundance of naturally-produced coho salmon (Oncorhynchus kisutch) on the Oregon Coast generally declined until these fish were listed as threatened under the Endangered Species Act in 1998. The subsequent rebuilding of Oregon coastal coho adult abundance is coincident with increased habitat restoration, reduced hatchery production, and reduced harvest. Importantly, ocean survival also improved, thereby complicating the assessment of management effectiveness at the adult life stage. Our objective was to assess change in the freshwater production of juveniles (smolts) through time in order to determine if recent increases in adult abundance could be related to management affecting the freshwater juvenile production.

Table 1. Six different three-variable generalized additive models were identified by Rupp et al. (2012b) to predict coho ocean abundance. These models were refitted with smolt-to-adult survival as the response variable. The annual smolt-to-adult survival prediction is an average of the logit transformed values predicted by each of the six generalized additive models. Variables used in the models are four-year moving average of Pacific decadal oscillation index (PDO), date of spring transition between down-welling and upwelling (SPR), upwelling index (UWI), sea surface height (SSH), spawner abundance (SPN), Multivariate El Niño-Southern Oscillation index (MEI), and sea surface temperature (SST). The three letters following the dot give the three consecutive months over which the variable was measured. The coefficient of determination $\left(R^{2}\right)$ and ordinary cross validation $(\mathrm{OCV})$ are given for individual models and the model averaged ensemble.

\begin{tabular}{llllll}
\hline ID & & Variables & & R$^{2}$ & OCV \\
\hline Model 1 & PDO.MJJ4 $_{\text {Model 2 }}$ & SPR & SPN & 0.71 & 0.36 \\
Model 3 & PDO.MJJ $_{4}$ & MEI.OND & UWI.JAS & 0.57 & 0.07 \\
Model 4 & PDO.MJJ & SPR & MEI.OND & 0.68 & 0.45 \\
Model 5 & PDO.MJJ & SWI.JAS & SST.AMJ & 0.61 & 0.24 \\
Model 6 & PDO.MJJ & SSH.AMJ & UWI.JAS & 0.77 & 0.50 \\
Models 1 - 6 ensemble & & SST.J & 0.74 & 0.46 \\
\hline \hline
\end{tabular}

Table 2. Three models of Oregon coastal coho population dynamics (columns) are distinguished from one another by whether or not (i) the relative reproductive success (RRS) of hatchery-origin fish is estimated, and (ii) a quadratic function of freshwater stream flow is used as a covariate in smolt recruitment. All three models permitted change in the mean, across-population inflection point in coastal coho smolt recruitment before $\left(\bar{\mu}_{\gamma 1}\right)$ and after $\left(\bar{\mu}_{\gamma 2}\right)$ 1998. The probability (Pr) that smolt recruitment declined was found by conducting random permutations of the posterior distributions. The odds ratio for improved recruitment is computed directly from the associated probabilities.

\begin{tabular}{llll}
\hline Model ID & A & B & C \\
\hline Change recruitment? & Yes & Yes & Yes \\
Estimate RRS? & Yes & No & No \\
Include Flow? & Yes & Yes & No \\
$\bar{\mu}_{\gamma 1}$ & 623 & 657 & 656 \\
$\bar{\mu}_{\gamma 2}$ & 407 & 434 & 434 \\
$\operatorname{Pr}\left[\bar{\mu}_{\gamma 1}>\bar{\mu}_{\gamma 2}\right]$ & 0.87 & 0.86 & 0.86 \\
Oddsratio $\bar{\mu}_{\gamma 2}>\bar{\mu}_{\gamma 1}$ & 1 in 6.8 & 1 in 6.2 & 1 in 6.1 \\
\hline \hline
\end{tabular}

All correspondence should be addressed to M. Falcy. 
We combined 46 years of data associated with 18 populations of Oregon coastal coho. Spawner-to-smolt relationships were modeled with Bayesian hierarchical state-space implementations of the logistic hockey stick recruitment function. We also develop a method of estimating the relative reproductive success of hatchery spawners. We found more evidence for decline than increase in productivity in the spawner-to-smolt life stage, suggesting that changes in physical oceanographic conditions are responsible for recent increases in adult abundance. The reproductive success of hatchery-origin fish relative to natural-origin fish was 0.51 with a $95 \%$ credible interval from 0.19 to 0.89 . While some management effects may unfold on longer time-scales than we observed, we nonetheless contend that carefully tailored models of non-stationary population dynamics are needed to understand and the effectiveness of management actions intended to recover populations.
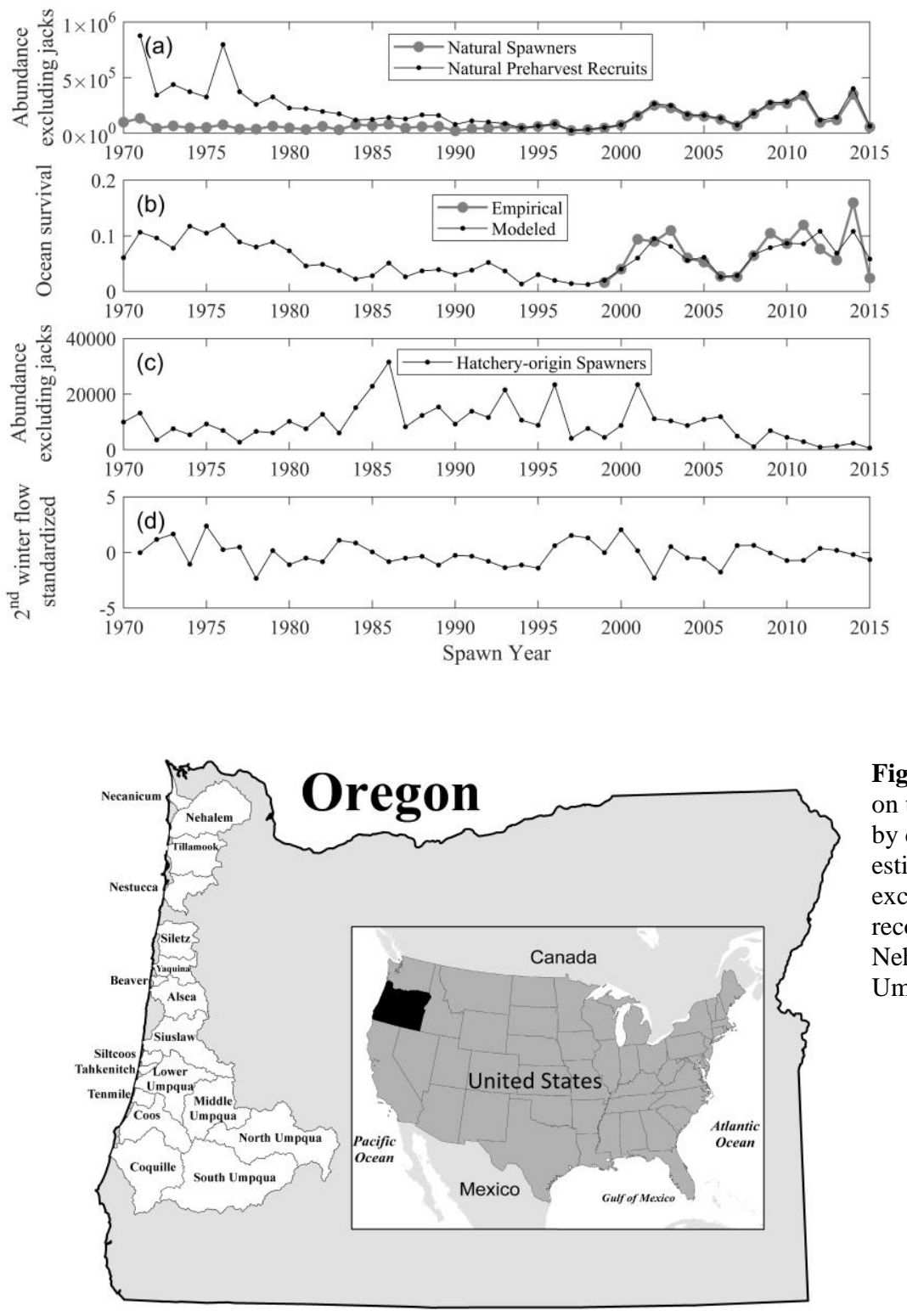

Fig. 1. The abundance of naturalorigin adult coho declined until the late 1990s (a), which is coincident with the pattern in ocean survival rate (b), but multiple biologically conservative actions also began in the late 1990s, including reduction in hatchery production (c). Mean second winter stream flow from November-March in four sites were standardized (z-score) over 19612015 and averaged together (d).
Fig. 2. Eighteen populations of coho salmon on the west coast of Oregon, USA, are defined by drainage basins. Abundance has been estimated annually since 1970 (see text for exceptions). Smolt-to-adult survival has been recorded since 1998 at sites located within Nehalem, Siletz, Yaquina, Alsea, Lower Umpqua, and Coos. 


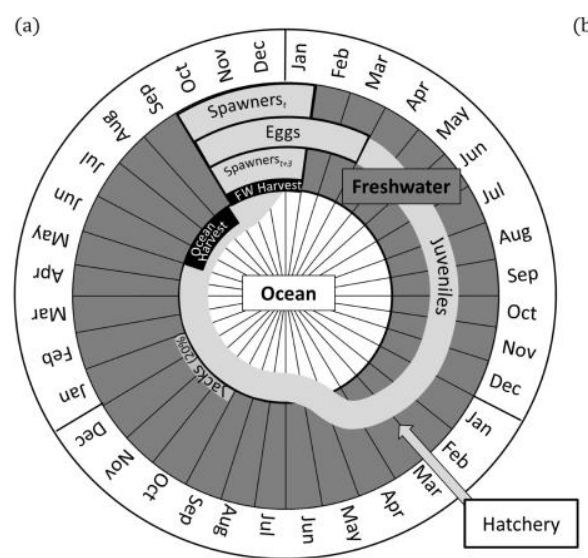

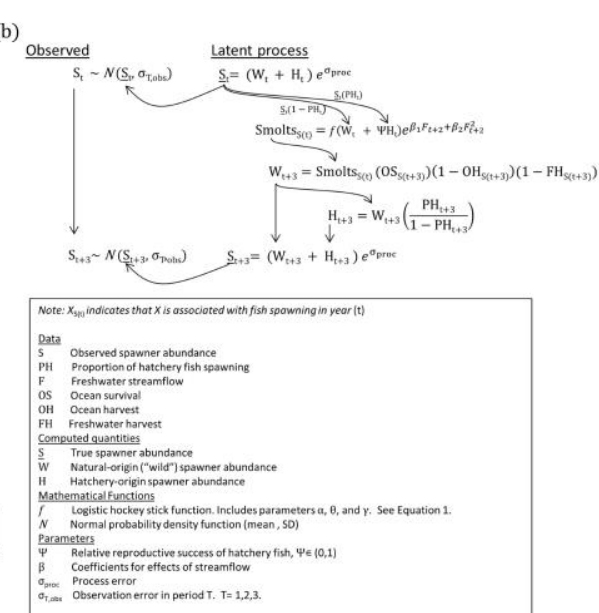

Fig. 3. Life cycle of Oregon coastal coho (a). Jacks are precocious males and not included in the data or models presented here. The statespace model (b) of coho population dynamics decomposes spawner abundance into hatchery and natural-origin components using annual estimates of the proportion of hatchery fish. Hatchery and natural-origin fish produce smolts, but reproductive success of hatchery fish is some fraction $(\Psi)$ of natural-origin fish that can be estimated empirically.

The latent process generating spawner abundance is recursive, but hatchery-origin fish are added to match the observed fraction of hatchery-origin spawners. Process error is logarithmic, reflecting the common assumption that recruitment is a multiplicative survival process. Each year's estimate of spawner abundance is assumed to be normally distributed around the true abundances, reflecting unbiased sampling error. The magnitude of the sampling error is allowed to vary across three time periods when different sampling designs were used. The parameter of the recruitment function controlling the inflection point $(\gamma)$ was allowed to vary across two periods (before and after 1998) that reflect times of pre- and post-biological conservation management.
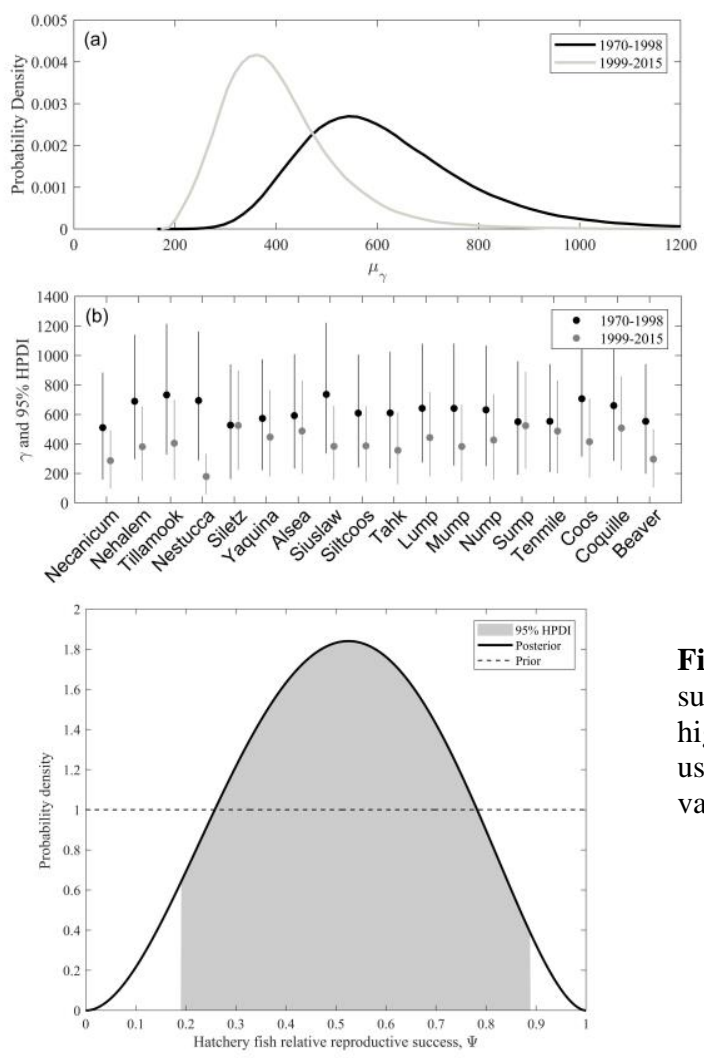

Fig. 4. Posterior probability distribution of the mean, allpopulation distribution of inflection points $(\gamma)$ in logistic hockey stick smolt recruitment functions from 1970 to 1998 and 1999 to 2015 (a). Individual population point estimates and 95\% highest probability density intervals for inflection points $(\gamma)$ in logistic hockey stick smolt recruitment functions from 1970 to 1998 and 1999 to 2015 (b). Model A used in both (a) and (b).
Fig. 5. Posterior probability density of hatchery fish reproductive success relative to natural-origin fish (solid line). Shaded area is the 95\% highest probability density interval $[0.19,0.89]$. The prior distribution used in the analysis is equi-probable over the entire range of possible values for hatchery fish relative reproductive success (dashed line).

\section{REFERENCES}

Falcy, M.R., and E. Suring. 2018. Detecting the effects of management regime shifts in dynamic environments using multi-population state-space models. Biol. Conserv. 221: 34-43. 